\title{
Antineutrophil antibodies associated with ulcerative colitis interact with the antigen(s) during the process of apoptosis
}

\author{
J Mallolas, M Esteve, E Rius, E Cabré, M A Gassull
}

\begin{abstract}
Background-Cell death by apoptosis seems to be an important mechanism for translocation to the cell surface of a variety of intracellular components capable of inducing autoantibody production. Aims-To identify the cellular location of antigen (Ag)-antineutrophil cytoplasmic antibodies (ANCA) in non-apoptotic human neutrophils, and to assess if ANCA associated with ulcerative colitis reacts with neutrophil antigen(s) during neutrophil apoptosis. The cellular distribution of Ag-ANCA in apoptotic neutrophils was also investigated.

Methods-Sera from 18 ulcerative colitis patients known to be positive for perinuclear IgG-ANCA (titre $\geqslant 1 / 320$ ), as assessed by indirect immunofluorescence (IIF), were analysed by immunofluorescent confocal laser scanning microscopy. ANCA were identified with fluorescein isothiocyanate (FITC) and tetramethylrhodamine isothiocyanate (TRITC) in non-apoptotic and apoptotic neutrophils, respectively. Apoptotic and non-apoptotic DNA was labelled with FITC and propidium iodide, respectively. Cycloheximide was added to polymorphonuclear leucocyte culture to induce apoptosis.
\end{abstract}

Results-Three patterns of scanning laser immunofluorescence microscopy in nonapoptotic neutrophils were observed with respect to cellular ulcerative colitis associated ANCA distribution: (1) diffuse nuclear localisation (16.7\%); (2) nuclear localisation in the nuclear periphery (50\%); and (3) mixed nuclear and cytoplasmic localisation $(33.4 \%)$. In all sera ANCA fluorescence colocalised almost completely with apoptotic DNA, with persistence of a diffuse and intense fluorescence. No significant changes in ANCA titres were found in non-apoptotic neutrophils.

Conclusions-The antigen(s) of ANCA associated with ulcerative colitis seems to be localised in most cases in the neutrophil nucleus. The almost identical colocalisation of ANCA and apoptotic cleaved DNA suggests that intracellular DNA redistribution during neutrophil apoptosis may play a role in antigen exposure to the immune system and ANCA production in ulcerative colitis.

(Gut 2000;47:74-78)
Keywords: antineutrophil cytoplasmic antibody; antigen; ulcerative colitis; apoptosis; humoral immunity; immunofluorescent laser confocal microscopy

The pathogenic importance of antineutrophil cytoplasmic antibodies (ANCA) associated with ulcerative colitis (UC) is controversial as both the antigen responsible for their production and how the antigen-antibody interaction occurs in vivo are unknown. A number of nuclear $^{1-3}$ and cytoplasmic antigens ${ }^{3-5}$ have been proposed as a possible target for ANCA in UC. However, none has been proved to account for ANCA production in all patients with UC.

In contrast, it is not known how the antigenic intracellular constituents are accessible to the immune system to trigger ANCA production and how they interact. These mechanisms are better known in Wegener's granulomatosis where activated neutrophils have been demonstrated to express ANCA target antigens on their surface making them accessible to these antibodies. "In vitro", such an interaction has been shown to result in neutrophil death by necrosis in the setting of an increased oxidative burst. $^{6}$ In contrast, UC associated ANCA (UC-ANCA) have no effect on free radical neutrophil production ${ }^{7}$ suggesting that mechanisms other than priming neutrophils may be involved in the Ag-ANCA interaction in UC. Consequently, it has been demonstrated that apoptosis of non-primed neutrophils may be an important mechanism for the interaction of ANCA directed against myeloperoxidase in systemic vasculitis. ${ }^{8}$

The aims of this study were: (1) to identify the cellular location of antigen-ANCA in nonapoptotic human neutrophils; and (2) to assess if ANCA associated with UC react with neutrophil antigen(s) during neutrophil apoptosis. We also determined the cellular distribution of Ag-ANCA in apoptotic neutrophils.

Abbreviations used in this paper: ANCA, antineutrophil cytoplasmic antibodies; ANA, antinuclear antibodies; UC, ulcerative colitis; SLE, systemic lupus erythematosus; IIF, indirect immunofluorescence; Ag, antigen; UC-ANCA, ANCA associated UC; PMN, polymorphonuclear leucocytes; FITC, fluorescein isothiocyanate; TRITC, tetramethylrhodamine isothiocyanate; PI, propidium iodide; TdT, terminal deoxynucleotydil transferase; TUNEL, TdT mediated fluorescein nucleotides (FITC-dUTP) nick end labelling. 


\section{Patients and methods}

PATIENTS AND CONTROLS

Sera from 18 UC patients were selected from a serum bank fulfilling the following criteria: positive for IgG-ANCA with a titre $\geqslant 1 / 320$; a clear ANCA perinuclear pattern; and negative for antinuclear antibodies (ANA) tested with HEp-2 cells and rat liver sections using an indirect immunofluorescence (IIF) assay. A diagnosis of UC was established using the Lennard-Jones clinicopathological criteria. ${ }^{9}$ Seven patients had active and two inactive disease, as assessed by the Truelove index. ${ }^{10}$ The remaining nine patients had been colectomised at least two years before sampling. Four had total proctocolectomy with Brooke's ileostomy, and an ileal pouch anal anastomosis had been performed in five. Serum samples were stored at $-70^{\circ} \mathrm{C}$ until analysis. Sera from three healthy individuals, negative for both ANCA and ANA, were used as controls.

POLYMORPHONUCLEAR LEUCOCYTE ISOLATION Polymorphonuclear leucocytes (PMN) were isolated from peripheral blood of healthy volunteers using a standard technique with a solution containing sodium metrizoate and dextran 500 as previously described. ${ }^{11}$ The final suspension contained more than 95\% PMN, as counted on a haemocytometer.

PMN CULTURE AND INDUCTION OF APOPTOSIS Freshly isolated PMN $\left(2.5 \times 10^{6}\right.$ cells $\left./ \mathrm{ml}\right)$ were cultured and incubated for 18 hours at $37^{\circ} \mathrm{C}$ in a humidified atmosphere containing 5\% $\mathrm{CO}_{2}$ in RPMI 1640 supplemented with $10 \%$ heat inactivated fetal calf serum and antibiotics. Cycloheximide $(10 \mu \mathrm{g} / \mathrm{ml})$ was added to the medium to induce increased apoptosis. ${ }^{12} 13$ Apoptosis was demonstrated using three well established methods: (1) TUNEL [TdT mediated fluorescein nucleotides (FITC-dUTP) nick end labelling] (Boehringer Manheim, cat. No 1684 795), an in situ programmed cell death labelling method based on detection of DNA strand breaks labelling free 3'-OH termini with FITC-dUTP. Terminal deoxynucleotydil transferase (TdT) is the enzyme which catalyses polymerisation of modified nucleotides to free 3'-OH DNA ends. Incorporated fluorescein labelled dUTP was analysed under fluorescence and confocal laser scanning microscopy ${ }^{14}$; (2) detection of chromatin fragmentation into histone associated DNA fragments (mono- and oligonucleosomes). This gives a distinctive DNA ladder pattern on purification of lysed cells in $1 \%$ agarose gel electrophoresis ${ }^{15}$; and (3) analysis of cell morphology of cytocentrifuged preparations using methyl blue staining. ${ }^{15}$

ANCA DETECTION

Glass slides containing cytocentrifuged smears (Shandon Cytospin, Shandon Inc., Pittsburg, Pennsylvania, USA) of non-apoptotic and apoptotic human neutrophils from healthy volunteers (approximately 100000 per slide) were fixed in $100 \%$ ethanol at room temperature for five minutes and used as substrate. Fixed neutrophils were stored at $-20^{\circ} \mathrm{C}$ until use. Sera from UC ANCA positive patients and healthy controls were tested at $1 / 40$ dilution. Serum IgG-ANCA were visualised with either fluorescein isothiocyanate (FITC) or tetramethylrhodamine isothiocyanate (TRITC) conjugated rabbit antibodies against human IgG $(\gamma$ chains) (DAKO AS, F202, and R151, respectively) diluted $1 / 100$, and examined under fluorescence microscopy and confocal laser scanning microscopy (see below).

NON-APOPTOTIC DNA DETECTION

Propidium iodide (PI) staining $(5 \mu \mathrm{g} / \mathrm{ml} ; 10$ minutes; Sigma, St Louis, Missouri, USA) was used in ethanol fixed neutrophil slides to visualise nuclear DNA.

\section{APOPTOTIC DNA DETECTION}

The TUNEL technique was used to detect DNA breaks or nicks with labelled FITCdUTP in slides containing apoptotic neutrophils (see above).

INDIRECT IMMUNOFLUORESCENCE MICROSCOPY Slides containing non-apoptotic or apoptotic neutrophils were used. FITC conjugated rabbit antibodies against human IgG were used to visualise serum IgG-ANCA under fluorescence microscopy at 400× (Zeiss, Axioplan, Germany). Slides were assessed by two independent observers who were unaware of the patient's diagnosis. Control sera positive and negative for ANCA were included in each test batch. For each serum sample, ANCA titres using non-apoptotic and apoptotic neutrophils were quantified.

CONFOCAL LASER SCANNING MICROSCOPY To assess possible colocalisation of nuclear DNA/apoptotic DNA and ANCA, slides were examined after double immunofluorescence using confocal laser scanning microscopy. Confocal images were obtained with a Leica TCS NT scanning module (Leica Microsystems Heidelberg $\mathrm{GmbH}$, Germany) with a Kripton-Argon laser, coupled to a Leica DMRB microscope with $100 \times$ objective and using a $488 \mathrm{~nm}$ laser line and a 530/30 band pass filter for the FITC signal (channel 1) and $568 \mathrm{~nm}$ laser line and 590 long pass filter for the PI/TRITC signal (channel 2). Optical sectioning of neutrophils was performed in $500 \mathrm{~nm}$ steps and overlay images were recorded superimposing simultaneous images from each channel. Double staining was performed for ANCA (FITC) and nonapoptotic DNA (PI), and for ANCA (TRITC) and apoptotic DNA (FITC).

Colocalisation was identified in the overlay images by yellow staining caused by the mixture of the green signal from FITC and the red signal from TRITC or PI as follows: (1) colocalisation in non-apoptotic neutrophils was the result of superimposition of the green fluorescence of FITC labelled secondary antibodies which detect ANCA and the red signal of PI which stains DNA; (2) colocalisation in apoptotic neutrophils was the result of superimposition of the green fluorescence of FITCTUNEL which detects apoptotic DNA and the 

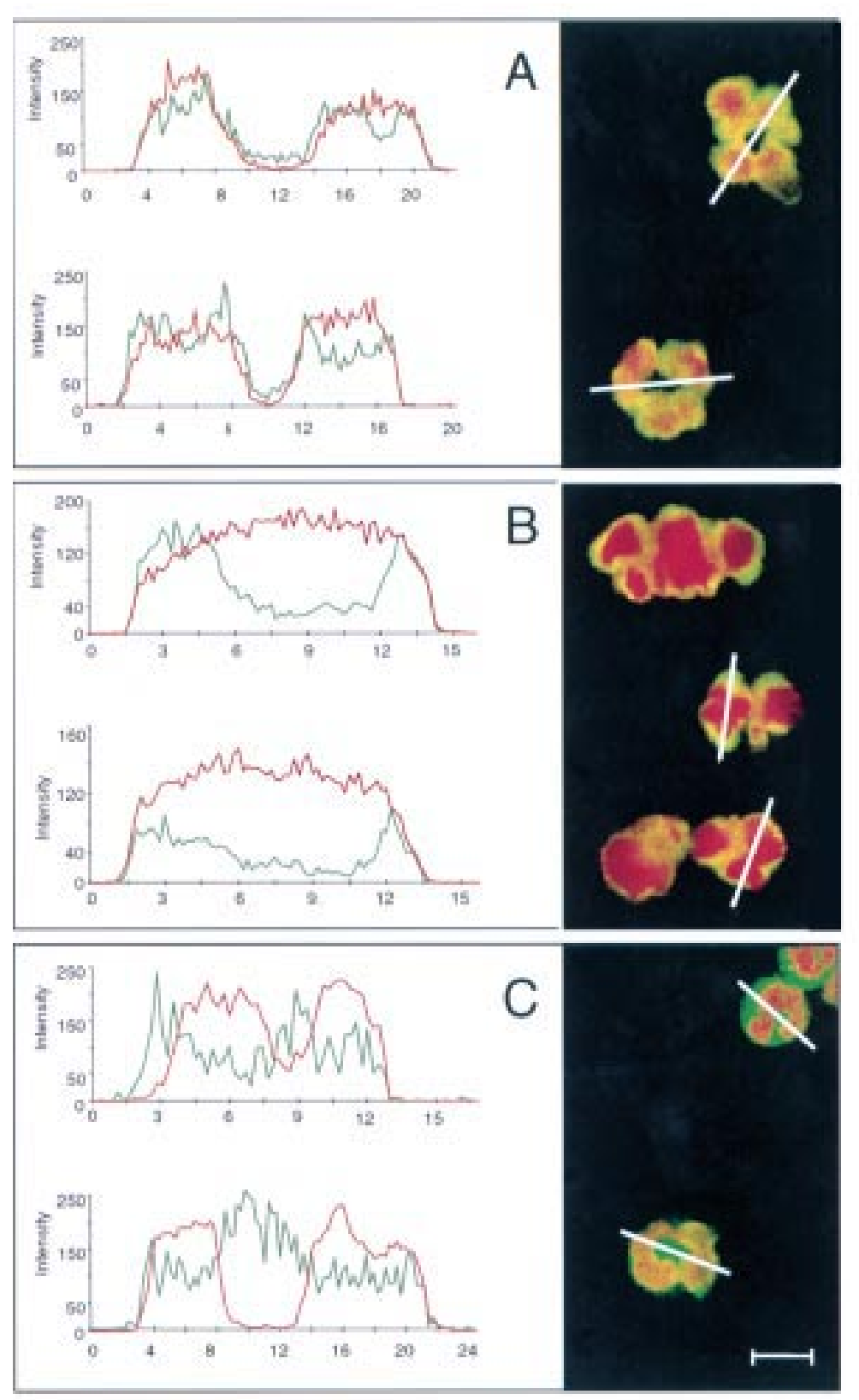

Xr. Pustion [jum]

Figure 1 Confocal laser scanning microscopy view of non-apoptotic ethanol fixed neutrophils. Antineutrophil cytoplasmic antibodies (ANCA) were detected with fluorescein isothiocyanate (FITC) conjugated secondary antibodies that produce a green signal at a wavelength of 500-560 $\mathrm{nm}$. DNA was visualised with propidium iodide which gives a red signal at wavelengths longer than $590 \mathrm{~nm}$. Three different patterns of ANCA localisation may be observed. (A) A diffuse nuclear ANCA associated ulcerative colitis (UC-ANCA) staining pattern, as demonstrated by yellow staining in the whole nuclear area as a result of superimposition of the two fluorescence signals. Complete overlay of the profile of staining intensity obtained by channel 1 (green profile, ANCA) and that obtained by channel 2 (red profile, DNA) may be observed. (B) Peripheral nuclear staining pattern, as demonstrated by a yellow rim-like ANCA fluorescence in the inner side of the nuclear periphery, overlapping with DNA. Yellow staining may be observed in the peripheral nuclear area as a result of superimposition of the two fluorescence signals. The profile obtained by channel 1 (green profile, $A N C A$ ) mainly localises in the inner side of the nucleus (channel 2, red profile, $D N A)$ periphery, as demonstrated by two peaks of greater intensity of ANCA staining in this location. (C) Mixed nuclear and cytoplasmic staining pattern. The green staining of the fluorescence signal of ANCA, non-superimposed with DNA, may be observed. The profile obtained by channel 1 (green profile, $A N C A$ ) is partly localised in the nucleus (channel 2, red profile, DNA) but overwhelms the DNA fluorescence boundary. Bar=10 $\mu \mathrm{m}$.

red signal of TRITC labelled secondary antibodies which detect ANCA. Yellow staining is more evident in those areas with similar immunofluorescence intensity for the two channels. In some areas of Ag-ANCA colocalisation with different immunoflorescence intensities, the red or green colour may predominate. Thus to give a more exact pattern of Ag-ANCA location, avoiding neutrophil image interpretation, colocalisation was also demonstrated graphically by a profile of fluorescence intensity obtained for each channel in a single line drawn through the cell sections.

\section{Results}

CONVENTIONAL INDIRECT IMMUNOFLUORESCENT ASSAY

On IIF examination with cytocentrifuged ethanol fixed non-apoptotic neutrophils, seven sera had an ANCA titre of $1 / 1280$, six had a titre of $1 / 640$, and five a titre of $1 / 320$. When retested using cytocentrifuged ethanol fixed apoptotic neutrophils, most sera also showed intense fluorescence with no significant changes with respect to the titres obtained with non-apoptotic neutrophils. The titres of only three sera decreased by two dilutions compared with the titre found with non-apoptotic neutrophils. In no case did an increase occur in ANCA titres observed using apoptotic neutrophils.

INDIRECT IMMUNOFLUORESCENT LASER

CONFOCAL MICROSCOPY

Non-apoptotic neutrophils

For cellular UC-ANCA distribution, three patterns of confocal laser immufluorescence were observed: (1) diffuse nuclear localisation, as illustrated by sera from three patients $(16.7 \%)$. In these cases yellow staining, corresponding to the overlap of the green ANCA fluorescence and the red DNA fluorescence, was observed in the whole nuclear area. The profile of the staining intensity obtained by channel 1 (green profile, ANCA) completely overlapped with that obtained by channel 2 (red profile, DNA) (fig 1A). In addition, the intensity of the ANCA fluorescence profile remained high in the entire area bounded by the DNA fluorescence profile; (2) peripheral nuclear localisation, as illustrated by sera from nine patients $(50 \%)$. In this pattern a yellow rim-like staining corresponding to the green ANCA fluorescence in the nuclear periphery overlapping with the red DNA fluorescence was observed. The profile obtained by ANCA staining (green profile) was mainly localised in the periphery of the nucleus as seen by two peaks of greater intensity of ANCA staining in this location (fig 1B); and (3) mixed nuclear and cytoplasmic localisation, as illustrated by the remaining sera from six subjects $(33.4 \%)$ (fig $1 \mathrm{C}$ ). In these cases the ANCA fluorescence profile (green profile) was partly superimposed on the DNA profile (red profile) but mainly exceeded the DNA boundary.

\section{Apoptotic neutrophils}

In all sera, ANCA fluorescence almost completely colocalised with apoptotic DNA and was observed for all three types of ANCA patterns. This may be seen as diffuse yellow staining as a result of ANCA rhodamine labelling and TUNEL fluorescein labelling superimposition 

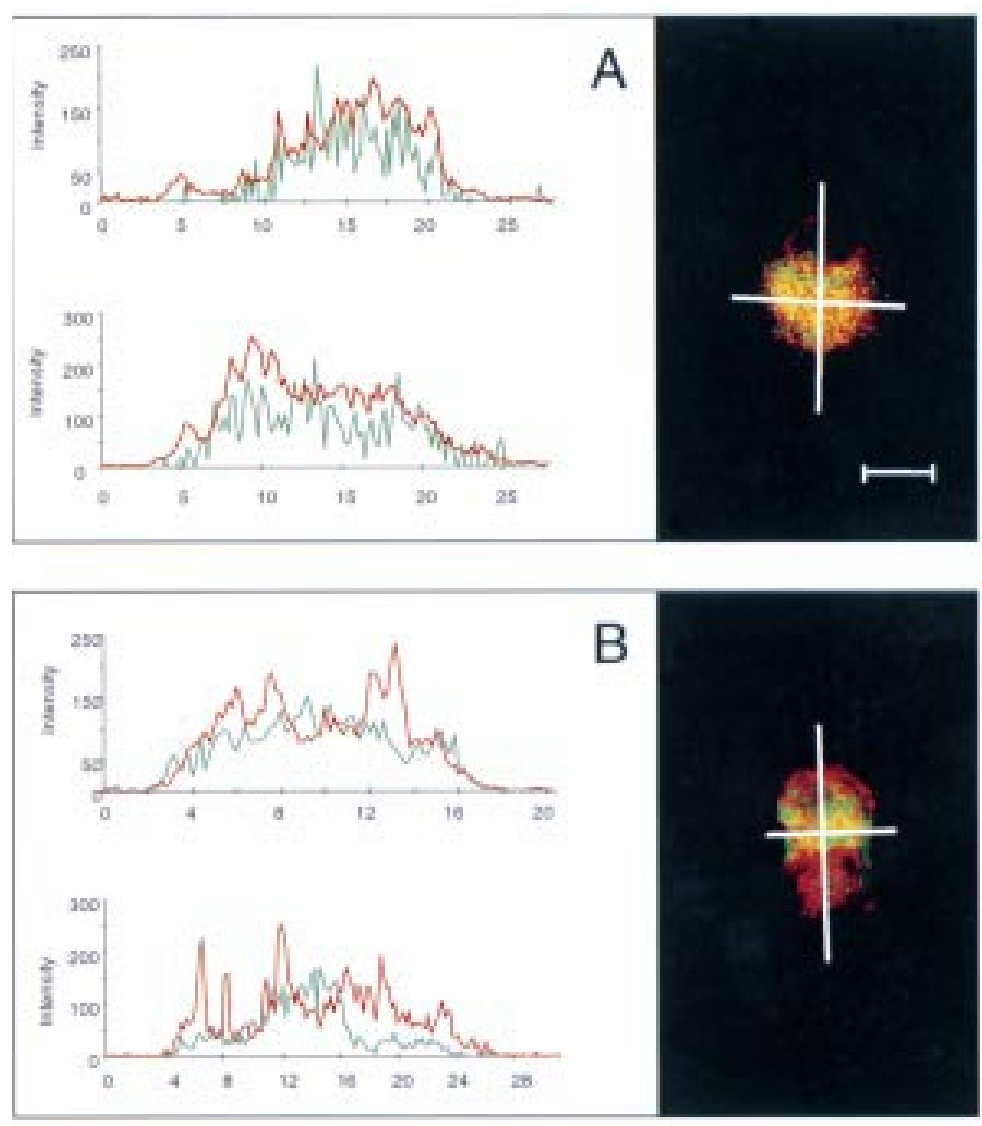

XYPoshon lim|

Figure 2 Confocal laser scanning microscopy of apoptotic ethanol fixed neutrophils. Antineutrophil cytoplasmic antibodies (ANCA) were detected with tetramethylrhodamine isothiocyanate (TRITC) which produces a red signal at wavelengths longer than $590 \mathrm{~nm}$. Apoptotic DNA was visualised with TUNEL (TdT mediated FITC-dUTP nick end labelling), an in situ programmed cell death labelling method based on detection of DNA strand breaks labelled with fluorescein isothiocyanate (FITC) which gives a green signal at wavelengths of 500-560 nm. (A) With most sera, ANCA staining showed diffuse

colocalisation with apoptotic cleaved DNA (oligonucleosomes and nucleosomes). Cross over fluorescence between FITC (cleaved DNA) and TRITC (ANCA) is shown by yellow staining. Almost complete superimposition of the profile of staining intensity obtained by channel 1 (red profile, ANCA) and that obtained by channel 2 (green profile, cleaved $D N A$ ) may be observed. (B) With three sera the ANCA staining intensity (red signal) was higher than that of apoptotic DNA (green signal) in some areas of the apoptotic neutrophils. These areas morphologically correspond to apoptotic blebs beyond the cytoplasmic membrane. The ANCA fluorescence profile (green profile) partly superimposed with the DNA profile (red profile) but exceeded the boundary of the apoptotic DNA. Bar=10 $\mu \mathrm{m}$.

(fig 2A). The ANCA profile (red profile) almost completely overlapped with the cleaved DNA profile (green profile). Only in three sera did some areas of the neutrophil show ANCA staining intensity higher than that of the apoptotic DNA. These areas seemed to correspond to apoptotic blebs which contained cleaved proteins and DNA. This occurred in some, but not all, neutrophils of the same slides (fig $2 \mathrm{~B}$ ).

\section{Discussion}

Humoral autoimmune phenomena are common in UC but the relevance of autoantibodies, particularly ANCA, in the pathogenesis of the disease remains to be established. ${ }^{16} \mathrm{UC}$ ANCA have not been shown to impair neutrophil function, ${ }^{7}$ their presence is not related to the severity or extent of the disease, ${ }^{3}{ }^{17} 18$ and in a high percentage of patients they may remain for years after colectomy. ${ }^{18}$ This evidence argues against a decisive role of UC-ANCA in tissue damage.

In the present study immunodetection of UC-ANCA during neutrophil apoptosis was investigated as UC-ANCA do not appear to induce neutrophil necrosis in UC patients. ${ }^{7}$ This is an important aspect as, in contrast with necrosis, cell death by apoptosis is not accompanied by release of proinflammatory mediators, ${ }^{19}$ thus limiting the degree of tissue injury. In contrast, apoptosis seems to be an important mechanism for translocation of a variety of intracellular components to the cell surface and their subsequent exposure to the immune system to induce autoantibody production. ${ }^{8}$ Hence it has been demonstrated that apoptotic cells are a major source of immunogen material in ANCA associated vasculitis $^{8}$ and other autoimmune diseases. ${ }^{20}$ An example of these apoptotic antigens are the nucleosomal DNA-histone complexes produced as a result of internucleosomal cleavage during apoptosis in systemic lupus erythematosus (SLE). ${ }^{21}$ In the present study we have demonstrated, using both IIF and indirect immunofluorescent confocal laser microscopy, that the antigen(s) associated with UC-ANCA remains viable during the apoptotic process of neutrophils, as the Ag-ANCA reaction is maintained. On laser confocal microscopy, apoptotic neutrophils show the typical apoptotic blebs due to chromatin crumpling and DNA reorganisation. In all sera, ANCA and cleaved DNA colocalised almost completely, suggesting that UC-ANCA could be directed against chromatin (packages of nucleosomes connected by DNA linkers) or nucleosomes (166240 base pairs of DNA wound around several types of histones) rather than against a specific protein. Although a number of nuclear proteins, such as histone $\mathrm{H} 1.3,{ }^{2}$ lamina proteins, ${ }^{3}$ and $\mathrm{HMG}$ proteins ${ }^{1}$ have been proposed as a target antigen for UC-ANCA, ANCA production would be more conceivably induced by DNA-protein complexes than by protein free DNA. Such an effect has been demonstrated for anti-DNA antibody production in animal model $^{22}$ and in patients with SLE. ${ }^{23} 24$

Immunodetection of the antigen(s) could theoretically increase during neutrophil apoptosis as changes in DNA structure may allow accessibility to otherwise hidden epitopes. Such a phenomenon has been demonstrated for the histone $\mathrm{H} 2 \mathrm{~B}$ during the early stages of apoptosis in activated lymphocytes. ${ }^{25}$ In the present study, however, we found no such increase as significant changes in the intensity of the immunofluorescence in apoptotic neutrophils did not occur. Moreover, when ANCA titres using apoptotic neutrophils were compared with those obtained using non-apoptotic neutrophils as substrate, a slight decrease in ANCA titres was observed in three sera. This suggests that total antigen amount either slightly diminished or was diffusely redistributed within the cell during apoptosis.

Further studies should clarify if the ability of UC-ANCA to engage apoptotic neutrophils might facilitate its removal by phagocytes without inducing free radical production and thus 
limiting the degree of tissue injury. The protective effect of autoantibodies has been suggested by experiments that crossed T cell receptor $\alpha$ deficient mice with an immunoglobulin $\mu$ deficient mice. In this experimental model, lack of immunoglobulins and presumably autoantibodies was associated with an increased incidence of colitis. ${ }^{26}$ The number of apoptotic cells in the epithelium and lamina propria of the colonic mucosa was markedly increased due to alteration in their clearance. ${ }^{26}$ Thus the occurrence of ANCA might not represent a deleterious but rather a protective effect as part of the normal immune homeostasis.

We also found that the antigen of UCANCA was closely related to DNA in nonapoptotic neutrophils as previously described. ${ }^{32}$ However, the staining pattern obtained by confocal laser microscopy differed from that described by Terjung and colleagues ${ }^{3}$ in which all UC related ANCA exhibited the so-called pattern III of ANCA staining. This consists of homogeneous rim-like ANCA fluorescence in the nuclear periphery partially overlapping with DNA. Such a pattern was observed in only $50 \%$ of the sera assessed in our study. In fact, we found three different patterns of UC related ANCA. Two showed nuclear localisation of the antigen: the diffuse nuclear and perinuclear patterns (in the inner side of the nucleus), and the other showed a mixed (nuclear and cytoplasmic) pattern. In this sense, our results resemble those of Billing et al in which $88 \%$ of UC p-ANCA positive sera showed nuclear localisation (perinuclear or diffuse).${ }^{27}$ The discrepancies could be due to differences in the method of handling neutrophils when preparing slides and have to be taken into account when assessing ANCA fluorescence patterns. We found no major differences in the morphology of neutrophils or variations in ANCA patterns using slides prepared at different cytocentrifuge speeds (200, 500, and $2000 \mathrm{rpm}$ ), different times (5 and 10 minutes), or different temperatures (4 and $22^{\circ} \mathrm{C}$ ) during ethanol fixation (data not shown).

In summary, the antigen of ANCA seems to be located mainly in the neutrophil nucleus. The almost complete colocalisation of UCANCA and cleaved DNA suggests that intracellular DNA redistribution during neutrophil apoptosis may play a role in the antigen-ANCA interaction in UC.

We are grateful to Anna Bosch (Serveis Científico-Tècnics, University of Barcelona, Campus Medicina, IDIBAPS) for technical assistance in confocal microscopy.

1 Sobajima J, Ozaki S, Osakada F, et al. Novel autoantigens of perinuclear antineutrophil cytoplasmic antibodies ( $\mathrm{p}-$ ANCA) in ulcerative colitis: non-histone chromosoma proteins, HMG1 and HMG2. Clin Exp Immunol 1997;107: 135-40.
2 Cohavy O, Eggena MP, Parsehhian M, et al. Histone H1, a candidate pANCA antigen in ulcerative colitis. Gastroenterology 1997;112:A951.

3 Terjung B, Herzog V, Worman $\mathrm{H} \mathrm{J}$, et al. Atypical antineutrophil cytoplasmic antibodies with perinuclear fluorescence in chronic inflammatory bowel diseases and hepatobiliary disorders colocalise with nuclear lamina proteins. Hepatology 1998;28:332-40.

4 Walmsley RS, Zhao MH, Hamilton MI, et al. Antineutrophil cytoplasm antibodies against bactercicida/permeability increasing protein in inflammatory bowel disease. Gut 1997;40:105-9

5 Ellerbroek PM, Outkerk Pool M, Ridwan BU, et al. Neutrophil cytoplasmic antibodies (pANCA) in ulcerative colitis. 7 Clin Pathol 1994;47:257-62.

6 Porges AJ, Redecha PB, Kimberly WT, Csernok E, Gross WL, Kimberly RP. Anti-neutrophil cytoplasmic antibodies engage and activate human neutrophils via Fc $\gamma$ RIIa. 7 engage and activate human

7 Gionchetti P, Vecchi M, Rizzello F, et al. Lack of effect of antineutrophil cytoplasmic antibodies associated with ulcerative colitis on superoxide anion production from neutrophils. Gut 1997;40:102-4.

8 Gilligan HM, Bredy B, Brady HR, et al. Antineutrophil cytoplasmic autoantibodies interact with primary granule constituents on the surface of apoptotic neutrophils in the absence of neutrophil priming. f Exp Med 1996;184:223141 .

9 Lennard-Jones JE. Classification of inflammatory bowel disease. Scand f Gastroenterol 1989;24(suppl 170):2-6.

10 Truelove SC, Witts LJ. Cortisone in ulcerative colitis. Report on therapeutic trial. BMF 1955;2:1041-6.

11 Bøyum A. Separation of white blood cells. Nature 1964;204: 793-7.

12 Tsuchida H, Takeda Y, Takei H, Shinzawa H, Takahashi T, Sendo F. In vivo regulation of rat neutrophil apoptosis occurring spontaneously or induced with TNF-alpha or cycloheximide. F Immunol 1995;154:2403-12.

13 Payne CM, Glasser L, Tischler ME, et al. Programmed cell death of the normal human neutrophil: an in vitro model of senescence. Microsc Res Tech 1994;28:327-44.

14 Gavrieli Y, Sherman Y, Ben Sasson SA. Identification of programmed cell death in situ via specific labelling of nuclear DNA fragmentation. $\mathcal{F}$ Cell Biol 1992;119:493-501.

15 Savill JS, Willie AH, Henson JE, Walport MJ, Henson PM, Haslett C. Macrophage phagocytosis of aging neutrophils in inflammation. $\mathcal{f}$ Clin Invest 1989;83:865-75.

16 Fiocchi C. Inflammatory bowel disease: etiology and pathogenesis. Gastroenterology 1998;115:182-205.

17 Esteve M, Mallolas F, Klassen J, et al. Antineutrophil cytoplasmic antibodies in sera from colectomized ulcerative colitis patients and its relation to the presence of pouchitis. Gut 1996;38:894-8.

18 Oudkerk Pool M, Ellerbroek PM, Ridwan BU, et al. Serum antineutrophil cytoplasmic autoantibodies in inflammatory bowel disease are mainly associated with ulcerative colitis. A correlation study between perinuclear antineutrophil cytoplasmic autoantibodies and clinical parameters, medical and surgical treatment. Gut 1993;34:46-50.

19 Haslett C. Granulocyte apoptosis and inflammatory disease. Br Med Bull 1997;53:669-83.

20 Hanh BH. Mechanisms of disease: Antibodies to DNA. $N$ Engl f Med 1998;338:1359-68.

21 Tax WJM, Kramers C, Van Bruggen MCK, Berden JHM. Apoptosis, nucleosomes and nephritis in systemic lupus erythematosus. Kidney Int 1995;48:666-71.

22 Desai DD, Krishnan MR, Swindle JT, Marion TN. Antigen-specific induction of antibodies against native mammalian DNA in nonautoimmune mice. F Immunol 1993;151:1614-26.

23 Burlingame RW, Boey ML, Starkebaum G, Rubin RL. The central role of chromatin in autoimmune responses to histones and DNA in systemic lupus erythematosus. 7 Clin Invest 1994;94:184-92.

24 Suenaga R, Abdou NI. Anti-(DNA-Histone) antibodies in active lupus nephritis. $\mathcal{F}$ Rheumatol 1996;23:279-85.

25 Zunino SJ, Singh MK, Bass J, Picker Lj. Immunodetection of histone epitopes correlates with early stages of apoptosis in activated human peripheral T lymphocytes. Am f Pathol 1996;149:653-63.

26 Mizoguchi A, Mizoguchi E, Neal Smith RN, Preffer FI, Bhan AK. Suppressive role of B cells in chronic colitis of T cell receptor $\alpha$ mutant mice. F Exp Med 1997;186:1749-56.

27 Billing P, Tahir S, Calfin B, et al. Nuclear localisation of the antigen detected by ulcerative colitis-associated perinuclear antineutrophil cytoplasmic antibodies. Am $\mathcal{f}$ Pathol 1995; 147:979-87. 